\title{
Characterization and control of droplets optically trapped in air
}

\section{Conference Paper}

Author(s):

David, Grégory; Reich, Oliver; Diveky, Matus (D); Roy, Sandra; Parmentier, Evelyne A.; Cremer, Johannes (D); Esat, Kivanç (D); Signorell, Ruth (1)

Publication date:

2019

Permanent link:

https://doi.org/10.3929/ethz-b-000369311

Rights / license:

In Copyright - Non-Commercial Use Permitted

Originally published in:

11083, https://doi.org/10.1117/12.2528743

Funding acknowledgement:

172472 - Phase Transitions of Ultrafine Aerosol Particles: Condensation, Freezing, and Metal Formation in Confined Systems (SNF)

177479 - Fundamentals of Aerosol Photoacoustic Spectroscopy (SNF) 


\section{Characterization and control of droplets optically trapped in air}

Grégory David, Oliver Reich, Matúš E. Divéky, Sandra Roy, Evelyne A. Parmentier, et al.

Grégory David, Oliver Reich, Matúš E. Divéky, Sandra Roy, Evelyne A. Parmentier, Johannes W. Cremer, Kivanç Esat, Ruth Signorell, "Characterization and control of droplets optically trapped in air," Proc. SPIE 11083, Optical Trapping and Optical Micromanipulation XVI, 1108322 (9 September 2019); doi: 10.1117/12.2528743

Event: SPIE Nanoscience + Engineering, 2019, San Diego, California, United States 


\title{
Characterization and control of droplets optically trapped in air
}

\author{
Grégory David*, Oliver Reich, Matus E. Diveky, Sandra Roy, Evelyne A. Parmentier, Johannes W. \\ Cremer, Kivanç Esat, and Ruth Signorell \\ Department of Chemistry and Applied Biosciences, Laboratory of Physical Chemistry, ETH Zurich, \\ Vladimir-Prelog-Weg 2, CH-8093, Zurich, Switzerland \\ *gregory.david@phys.chem.ethz.ch
}

Keywords: aerosol particles, single particle, optical trapping, light scattering, size fitting, feedback control mechanism.

\begin{abstract}
In this contribution we present experiments used to control and characterize single optically trapped aerosol particles. These experiments include a counter-propagating optical tweezer, a feedback control mechanism to stabilize the particle in the trap and a two-angle optical scattering measurement to monitor the time-evolution of the particle size. Experimental setups and results are presented for these experiments.
\end{abstract}

\section{INTRODUCTION}

Aerosol particles are ubiquitous in the air indoor ${ }^{1}$ and outdoor ${ }^{2}$. They strongly influence the climate ${ }^{3}$ and human health ${ }^{4}$. However the importance of aerosol particles remains difficult to evaluate precisely because of their complexity. They show numerous physicochemical properties (size, shape, phase, chemical composition, viscosity, refractive index, $\mathrm{pH}$, mass accommodation coefficient) and they are involved in complex processes, such as evaporation and condensation of gaseous species $^{5-7}$, light scattering and absorption ${ }^{8,9}$, photochemistry ${ }^{6,10-12}$, phase transitions ${ }^{13-15}$ and phase separation ${ }^{16,17}$. Better understanding these processes is interesting for both applied and fundamental research. In addition, the pathways and kinetics of such processes can be very different for aerosol particles compared with deposited particles ${ }^{18}$ or bulk solutions ${ }^{12}$. Single particle studies have proven useful to better understand such processes by avoiding ensemble averaging. Identifying stochastic or intermittent processes, such as phase transitions ${ }^{15}$ or certain fluorescence processes ${ }^{19}$, would not be possible for a large ensemble of particles. A thorough characterization of particle properties is necessary to better understand the processes in which they are involved. Here the particle size is a key parameter affecting these processes in aerosol particles ${ }^{7}$, 17, 20-22. We are developing multiple experiments to better control and characterize a single particle optically trapped in air: - Counter propagating optical tweezer (CPT) used to isolate single particles in air.

- Feedback control mechanism to control the trapping position of single particles and to improve the confinement of trapped particles.

- Polarization-resolved two-dimensional angular optical scattering (TAOS) of the trapping laser to size particles.

- Broadband light scattering (BLS) to measure the particle size and refractive index.

- Raman scattering to characterize the chemical composition of the particle.

- Digital in-line holography to image the particle shape and motion.

- Photoacoustic measurements to determine the light absorption by particles.

In addition, these experiment can be coupled to obtain a more thorough characterization of single particles. The BLS ${ }^{15,23}$, Raman scattering ${ }^{15}$, photoacoustics ${ }^{7,20,24}$ and digital holography ${ }^{25,26}$ experiments have already been discussed in details elsewhere and will not be discussed further here. This paper explains the feedback control mechanism and the polarizationresolved TAOS measurements. Some results obtained with these techniques are presented.

\section{METHODS}

\subsection{Optical trapping}

Single aerosol particles are isolated in air by using a CPT ${ }^{20,23}$. The scheme of the CPT is shown in Figure 1-a. A continuous laser (Opus 532, $532 \mathrm{~nm}$, power between 100 and $3000 \mathrm{~mW}$ depending on the application) is passed through a Faraday isolator to avoid back reflections to the laser head and through an electro-optic modulator (EOM) to control the polarization of the laser beam. The beam is expanded by a factor of four with a two lens telescope and then split by a 
polarization beamsplitter cube (PBC) to obtain two counter-propagating Gaussian beams. The splitting of the laser power between the two trapping beams is controlled by modifying the polarization of the laser with the EOM. As explained further in Section 2.2, the EOM is controlled by a feedback loop to control the trapping position of single particles and to better confine them. Each counter-propagating beam is finally focused with an aspherical lens. The counter propagating beams are aligned to propagate along the same axis. The foci of the two beams are adjusted to be shifted with respect to each other by $\sim 100-200 \mu \mathrm{m}$ in the Z-direction (the direction of propagation of the laser beams, see Figure 1-a and 1-b). This shift creates a potential well in which a single particle is trapped. Trapped particles oscillate around their equilibrium position due to their Brownian motion. The amplitude of this oscillation depends on the quality of the CPT alignment and can reach up to a few tens of micrometers along the Z-axis (without feedback control of the particle). Such oscillations cannot be observed along X-and $\mathrm{Y}$-axis where particles are much better confined. Trapped particles are isolated in air in an environmental trapping cell where the composition and relative humidity $(\mathrm{RH})$ of the surrounding gas can be controlled. The surrounding gas is either nitrogen (Pangas, nitrogen 5.0) or synthetic air (Pangas, synthetic air 5.6) depending on the application. To control the RH in the trapping cell we mix a dry (RH $\sim \%$ ) and a humidified (RH 100\%) flow of the chosen gas. Liquid particles are generated from solutions with a nebulizer (PARI LC SPRINT Nebulizer) or an atomizer (TSI 3076 atomizer). For solid particles, a solution in which particles are suspended is atomized or nebulized. Examples of particles studied include solutions of aqueous salt $\left(\mathrm{NaCl}, \mathrm{K}_{2} \mathrm{CO}_{3}\right)$ or sugar (raffinose, sucrose), organic compounds (oleic acid, tetra-ethylene glycol (TEG), glycerol) and solid particles (polystyrene-latex spheres (PSL)). The plume of particles generated by the atomizer or nebulizer is directed to the trapping cell where some particles are pushed towards the trapping position by the two trapping beams. For liquid particles, the trapping process is usually accompanied by coagulation of several droplets to a larger one. The rest of the particles are flushed away with a gas flow.

\subsection{Feedback control mechanism for particle stabilization}

We developed a feedback loop controlling the power balance of the two trapping beams to control the position of the trapped particles and to improve the confinement of particles ${ }^{7}$. As illustrated in Figure 1-b and 1-c, the power balance of the two trapping beams can be used to control the trapping position of single particles. The equilibrium position of the particle is reached when the sum of the forces exerted on the trapped particle is zero. The force acting on the trapped particle along the Z-axis, the direction where the particle is the least stable ${ }^{23}$, is the so-called scattering force. The scattering force corresponds to a transfer of momentum from the incident light beam to the particle due to light scattering and absorption of the particle. The scattering force pushes the particle in the direction of light propagation. Hence the scattering forces of both trapping beams have opposite directions. The scattering force $\mathrm{F}$ arising from each laser beam can be expressed as ${ }^{8,9}$ :

$$
F=I\left(C_{e x t}-C_{s c a}<\cos \theta>\right) / c
$$

where $\mathrm{I}$ is the light intensity of the respective trapping beam at the particle location, $\mathrm{C}_{\mathrm{ext}}$ is the extinction cross-section of the particle, $\mathrm{C}_{\text {sca }}$ is the scattering cross-section of the particle, $<\cos \theta>$ is the asymmetry parameter of the particle ${ }^{8}$ (average cosine of the scattering angle), and $\mathrm{c}$ is the velocity of light. $\mathrm{F}$ depends only on properties of the particle $\left(\mathrm{C}_{\text {ext }}, \mathrm{C}_{\text {sca, }},<\cos \theta>\right)$ and the intensity at the particle's location. The equilibrium position of the trapped particle is where the intensities of both trapping beams impinging on the particle are the same. For trapping beams of the same power, the equilibrium position is in the middle of the two foci, as illustrated in Figure 1-b. However, if one of the trapping beams has higher power than the other, the particle will shift further away from the focus of this trapping beam and closer to the focus of the other trapping beam to find a new equilibrium position (Figure 1-c). This principle is used experimentally to control the position of the trapped particles. In addition, the Brownian motion of the particles is also counteracted. If the particle shifts away from the desired equilibrium position, the power of the beam propagating against the particle shift is increased to push the particle back. A quadrant photodiode (QPD) is used to measure the position of the particle along the Z-axis. The QPD signal is sent to an analog PID controller to generate the feedback signal. This feedback signal controls the EOM voltage and adjusts the power balance between the two trapping beams. This feedback control mechanism allows trapping of single particles at a reproducible position, avoids drifts of the trapping position and improves the confinement of the particle to better than within the amplitude of Brownian motion (translational stabilization along Z). Such feedback loop stabilization improves the accuracy and reliability of the characterization of the trapped particles. 


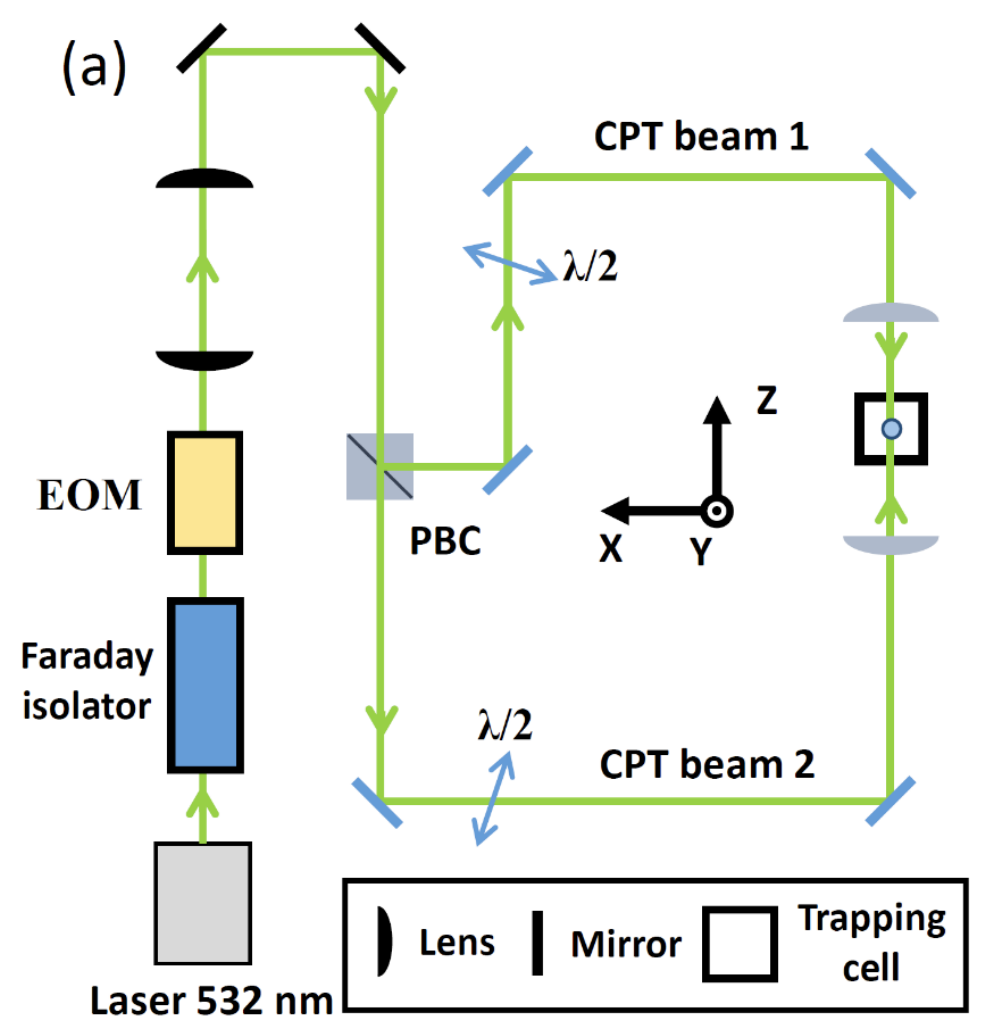

(b)

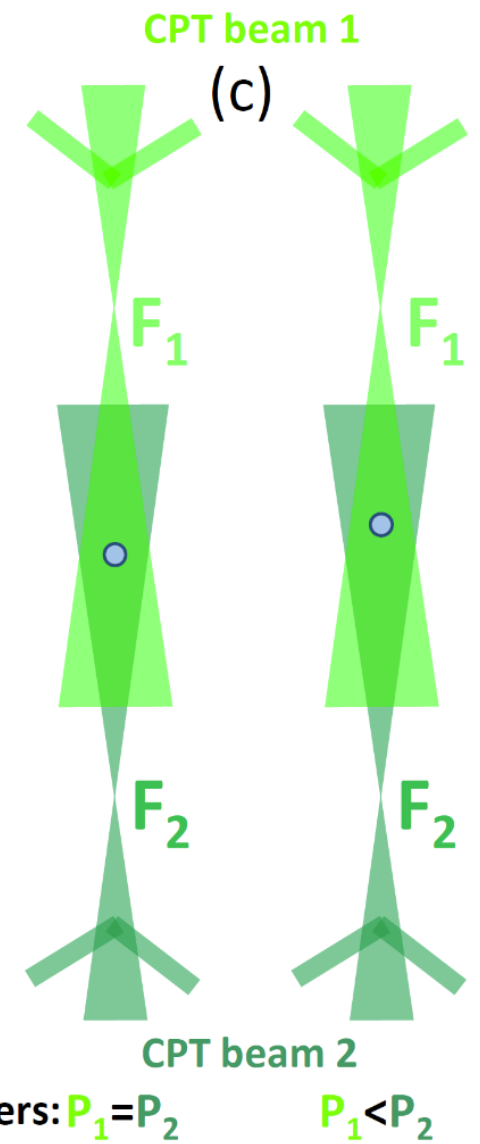

Figure 1. Scheme of the counter-propagating tweezer (CPT) (panel (a)). Half-wave plates ( $\lambda / 2)$ are used to control the polarization of the two trapping beams as desired (either cross-polarized or with the same polarization). Scheme of the particle equilibrium position as function of the balance power in the two CPT beams (panel (b) and (c)). Panel (b) shows the particle trapped in the middle of the two trapping beams when the two trapping beams have the same power. Panel (c) illustrates the situation for trapping beams with unequal power with the particle shifted from its equilibrium position towards the beam with lower power. $F_{1}$ and $F_{2}$ indicate the focus of the CPT beam 1 and 2 respectively.

\subsection{Size fitting with Total TAOS}

One way to retrieve the size of trapped single particles is to use the angular dependence of the elastic scattering of the particle at a fixed wavelength ${ }^{8,27}$. For this purpose the particle is illuminated with a single wavelength (trapping laser in this case) and the scattered light is imaged ${ }^{28}$ as illustrated in Figure 2. Such measurements are called two-dimensional angular optical scattering (TAOS) (one dimension corresponds to the scattering angle and the other to the azimuth angle of the particle scattering ${ }^{8}$ ). The TAOS pattern can be fitted with the appropriate scattering calculations to obtain the size of a particle for known particle shape, e.g. by using Mie theory for spherical particles. However, fitting the TAOS pattern is not very reliable for particles smaller than one or two micrometers in radius because the pattern does not contain sufficiently characteristic features to provide a unique solution for the fit. 


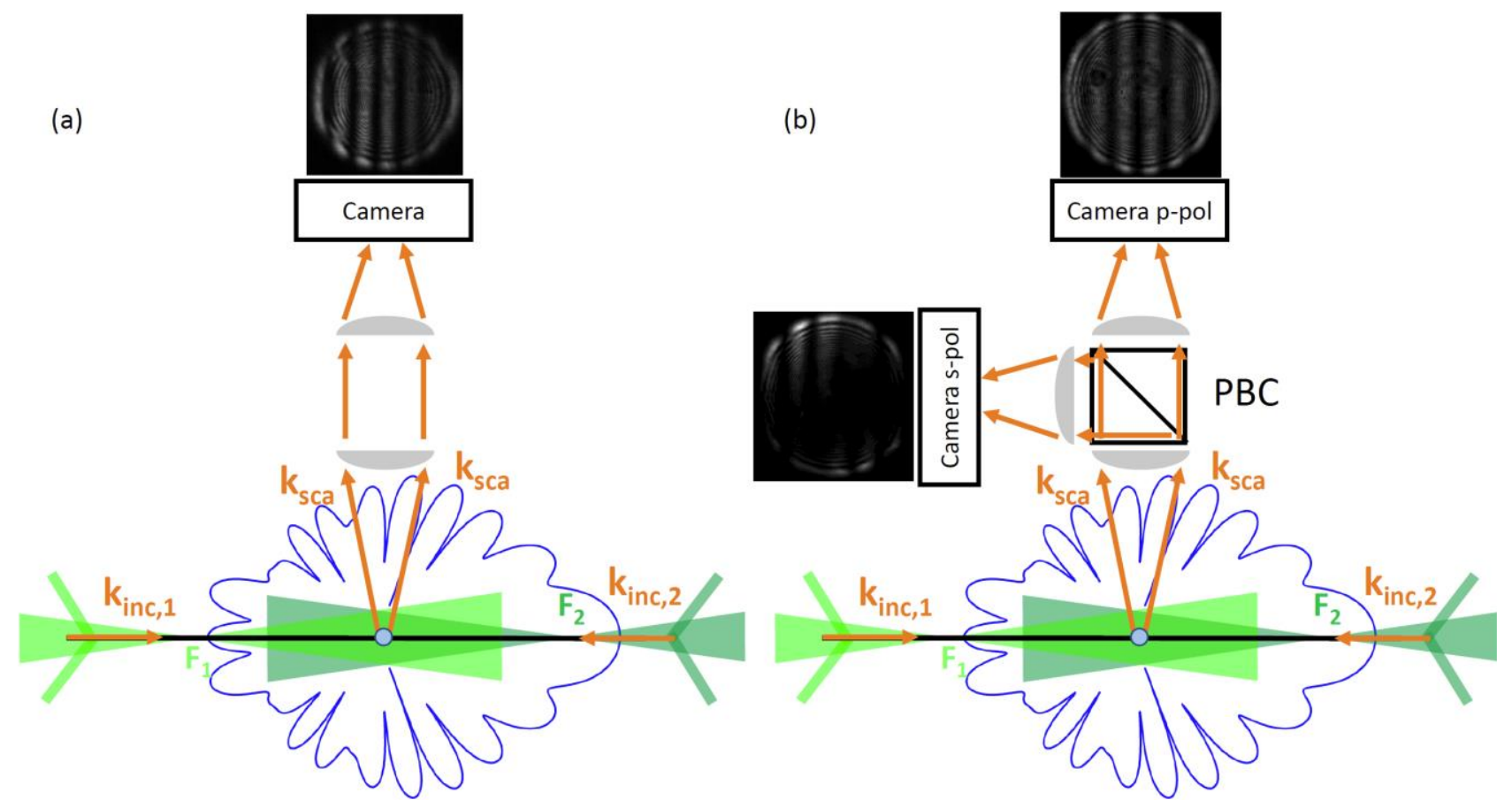

Figure 2. Scheme of TAOS measurements (not polarization-resolved (panel (a)) and polarization-resolved (panel (b))). The light scattered by the particle is collected and collimated with a lens or an objective. We use a microscope objective (Mitutoyo, 20x, numerical aperture 0.42 , working distance $20 \mathrm{~mm}$ ). A lens is then used to project an image of the scattered light onto the camera sensor. For the polarizationresolved TAOS measurements a $\mathrm{PBC}$ is used to separate the light scattering with p- and s-polarization with respect to the scattering plane. The scattering plane is the plane formed by the incident and scattered wave vector $\mathbf{k}_{\mathbf{i n c}}$ and $\mathbf{k}_{\text {sca }}$, respectively.

For measurements where the particle size changes over time, we developed a fit of the particle size using the total intensity of the TAOS (Total TAOS) to improve the reliability of particle-sizing with this technique. The Total TAOS is obtained by summing the counts of each pixel of the camera. When the particle size changes in time the Total TAOS also changes. Typical spectra obtained for the Total TAOS measurements are shown in Figure 3. For a spherical particle, the experimental Total TAOS is compared with Mie simulations for the size-dependent Total TAOS. When the refractive index of the particle is parameterized correctly, the peak shapes of the experimental data are reproduced in the Mie simulation, as shown in the Figure 3 andFigure A. 1. Two case studies are considered: photoacoustic spectroscopy of TEG particles (left column of Figure 3) and photochemistry of oleic acid particles (right column of Figure 3). The refractive index of TEG particles during the photoacoustic measurement is calculated by modeling the temperature of the particle and its water concentration as a function of the size of the particle ${ }^{7}$. Such size dependent refractive indices are necessary for a satisfactory fit of the Total TAOS for aqueous TEG particles. The refractive index of oleic acid particles is taken from previous BLS measurements of the oleic acid refractive index ${ }^{23}(\mathrm{~m}=1.48$ at $532 \mathrm{~nm})$. We tried to fit the time evolution of the particle refractive index but the best fits were found for constant refractive index throughout the whole Total TAOS fit. The refractive index of oleic acid particles is probably not changing significantly during photochemistry. When the appropriate refractive index of a particle is known, the time evolution of the particle size can then be retrieved by two different methods:

1) Assigning by eye each peak observed in the experimental Total TAOS to a peak in the simulated Total TAOS, as shown in Figure A. 1. As each peak in the simulated Total TAOS corresponds to a well-defined value of the particle radius this yields a time-series of particle radii (panel (c) of Figure A. 1), which can be fitted to a suitable empirical model function for the time evolution of the particle size. We found a double exponential decay for TEG particles ${ }^{7}$ (Equation 2 ) and a third order polynomial for the oleic acid particles (Equation 3) to provide the best fits of the respective experimental time series.

$$
\begin{aligned}
& R(t)=R_{0}+B_{1}\left(\exp \left(-\frac{t}{A_{1}}\right)-1\right)+B_{2}\left(\exp \left(-\frac{t}{A_{2}}\right)-1\right) \\
& R(t)=R_{0}+C_{1} t+C_{2} t^{2}+C_{3} t^{3}
\end{aligned}
$$


2) The second method of retrieving the time evolution of the particle size is a fully-automated fit. The appropriate empirical function, Equation (2) or (3) for our case studies, is used with a grid of values for each parameters to generate different possible time-evolutions of the particle radius. For each time-evolution of the particle size, the corresponding Total TAOS is simulated and compared to the experimental Total TAOS. The best fit parameters are found by minimization of the sum of squared deviations (SSQ) between the simulated and experimental Total TAOS. Four successive grid search loops are used in the fit to optimize the calculation time. Each successive loop uses the results of the previous one to refine the retrieved time evolution of the particle size. The two methods used to retrieve the time-evolution of the particle radius provide very similar results. Nonetheless the second method may provide more objective results as it is a fully-automated fit.

The polarization of the light scattered by the particle contains additional information that can be useful for particle sizing. As shown in the panel (b) of Figure 2, the Total TAOS pattern can be decomposed into two signals, one for parallel (ppol) and one for perpendicular polarization (s-pol) with respect to the scattering plane. Each Total TAOS is fitted by Mie simulations of the Total TAOS corresponding to the respective polarization. The fitting procedure of polarization-resolved Total TAOS is the same as explained before. The only difference is that for polarization-resolved Total TAOS two signals need to be fitted simultaneously for a given time-evolution of the particle. The use of polarization-resolved Total TAOS makes the of the particle size more reliable because each polarization has specific resonances with distinct line shapes.

\section{RESULTS AND DISCUSSION}

The implemented feedback control mechanism improved the trapping stability of single particles. Single particles are trapped at a reproducible position, without any temporal drift of the position. In addition, with the feedback control mechanism, no noticeable motion of the particle around its trapping position is observed. Such a confinement of the particle allows one to perform other measurements for particle characterization under optimal conditions.

\subsection{Size fitting of TEG and oleic acid particles}

Typical Total TAOS data for TEG and oleic acid particles are presented in Figure 3. Total TAOS measured on oleic acid particles are polarization-resolved while the measurements on TEG particles are not. The time evolutions of the particle radius $(\mathrm{R}(\mathrm{t}))$ for the TEG particle covers the range from $1870 \mathrm{~nm}$ to $750 \mathrm{~nm}$ in approximately 8 minutes. The fast shrinking of the particle is induced by the absorption of the IR laser, which increases the temperature of the particle and consequently results in enhanced evaporation. The shrinking of the oleic acid particle takes place over a longer time scale than that of TEG particles. The particle shrinks from $1870 \mathrm{~nm}$ to $1120 \mathrm{~nm}$ in approximately three hours. The size change of the oleic acid particle is due to a photochemical reaction induced by the trapping laser and will be discussed in a forthcoming paper. The retrieved $\mathrm{R}(\mathrm{t})$ for the oleic acid particle seems to depend almost linearly on time, but to obtain a satisfactory agreement between measurement and fit second and third order terms are necessary. The good agreement of the Total TAOS fit with the experimental Total TAOS confirms the validity of the refractive indices and the fit parameters derived for the time evolution observed in the respective experiments. 


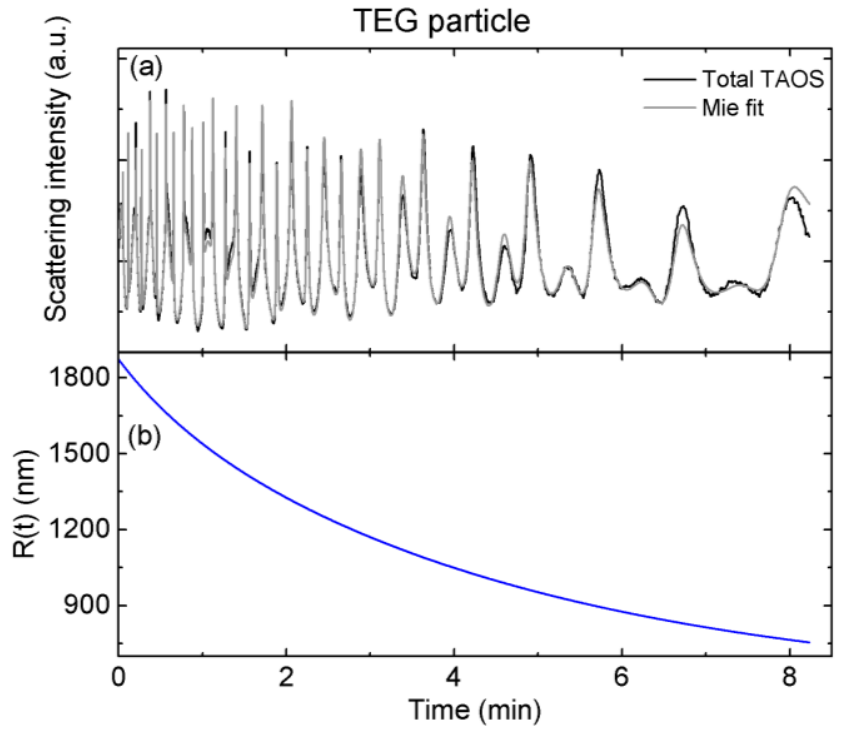

Figure 3 Typical Total TAOS for a TEG (left column) and an oleic acid particle (right column) together with corresponding fits and time evolutions of the particle radius $(\mathrm{R}(\mathrm{t})$ ). The measurement of the TEG particle is shown in black and the fit in grey (panels (a)). The Total TAOS of the TEG particle is not polarization-resolved, while the Total TAOS of the oleic particle is polarization-resolved. The spol Total TAOS measurement is shown in panels (c) and the p-pol Total TAOS measurement is plotted in panel (d). The retrieved R(t) are shown in panels (b) and (e).

As can be seen in Figure 4-a, the peak position of the experimental Total TAOS and the best fit do not match perfectly. Figure 4-a and -b correspond to the data in Figure 3-a, with the time dependence replaced by the size-dependence (the retrieved $\mathrm{R}(\mathrm{t})$ is used to convert the time axis into a size axis). The difference between the position of a measured peak and its fit define the fit error (see Figure 4-c). For most radii, the fit error is around $1 \mathrm{~nm}$, but it increases to a few nanometers for large and small radii. Such errors represent typical cases. However, when R(t) spans a very large size range, the error can also increase up to a few tens of nanometers. Errors of up to $50 \mathrm{~nm}$ were found at the very beginning of the measurement (i.e. for large sizes) for a TEG particle that shrinked from $3600 \mathrm{~nm}$ in radius down to $1360 \mathrm{~nm}$. However the error quickly drops below a couple of nanometers after this initial phase. Generally, Total TAOS measurements provide reliable way to determine the size of particles with radii in the range from a few hundreds of nanometers up to several micrometers. 

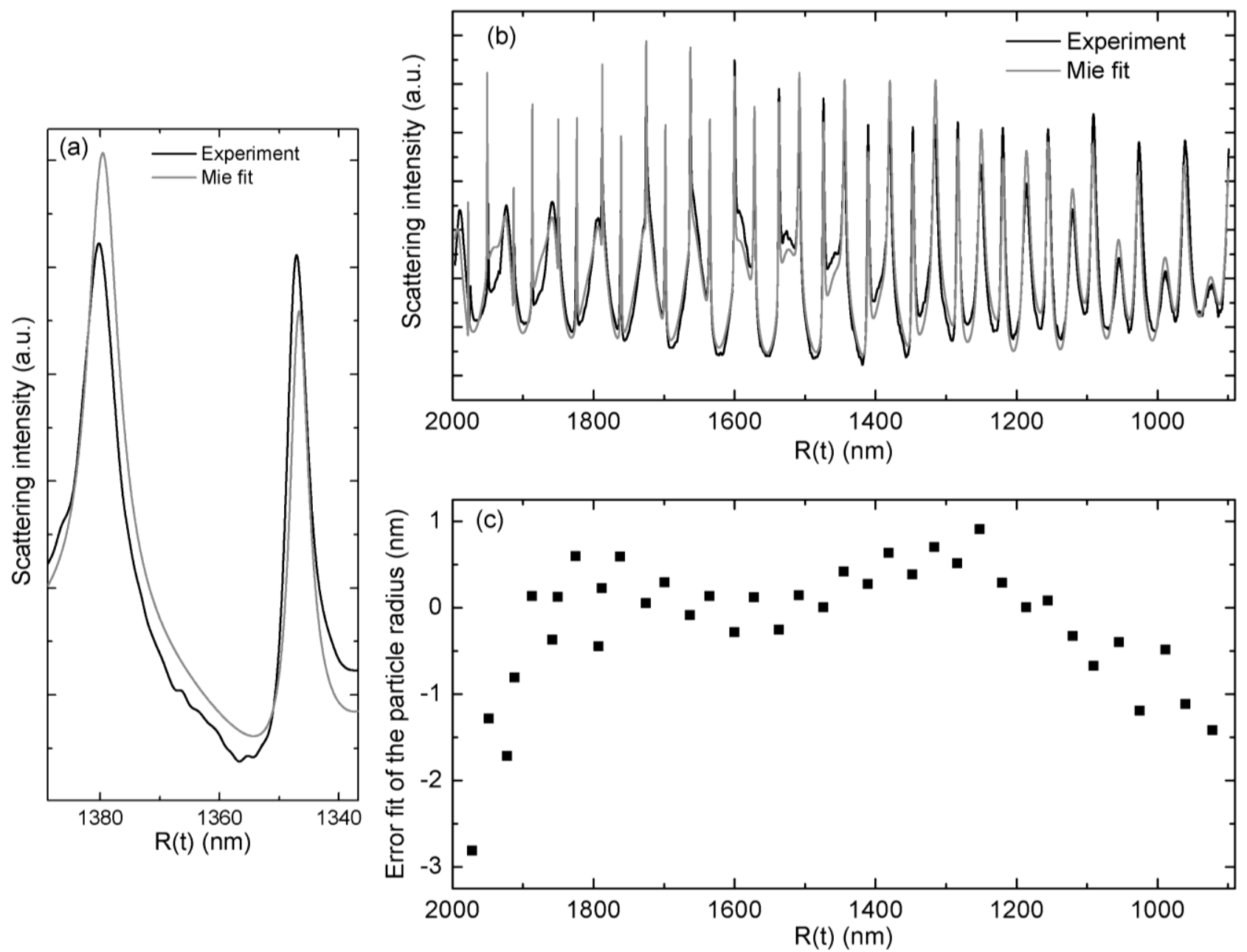

Figure 4. Quality of Total TAOS fitting. Zoom of two Total TAOS peaks where a slight shift and a miss-match of the peak amplitude between the measurement and the fit are observed (panel (a)). Survey of the time evolution of the measured and fitted Total TAOS as a function of the retrieved $\mathrm{R}(\mathrm{t})$ (panel (b)) for the same particle as in panel (a). Error retrieved from the deviation of peak positions in the measured and fitted Total TAOS (panel (c)).

\section{SUMMARY}

We have presented different methods to trap and characterize single aerosol particles. A feedback control mechanism has been developed to control the trapping position of the single particle; i.e. to avoid temporal drifts of the trapping position and to better confine the particle around the trapping position. The total intensity of two-dimensional angular optical scattering of a particle has been used to retrieve the temporal evolution of the particle size for evaporating droplets.

\section{APPENDIX A. DETAILS ABOUT SIZE FITTING WITH TOTAL TAOS}

Figure A.1 shows the assignment of the experimental Total TAOS peaks (a) to the simulated Total TAOS peaks (b). This assignment provides the conversion from a time scale to a radius scale (c), from which an empirical function can be extracted to describe the time evolution of the particle radius. This empirical function can then be used to perform fully automated fits of the time-evolution of the particle radius. 

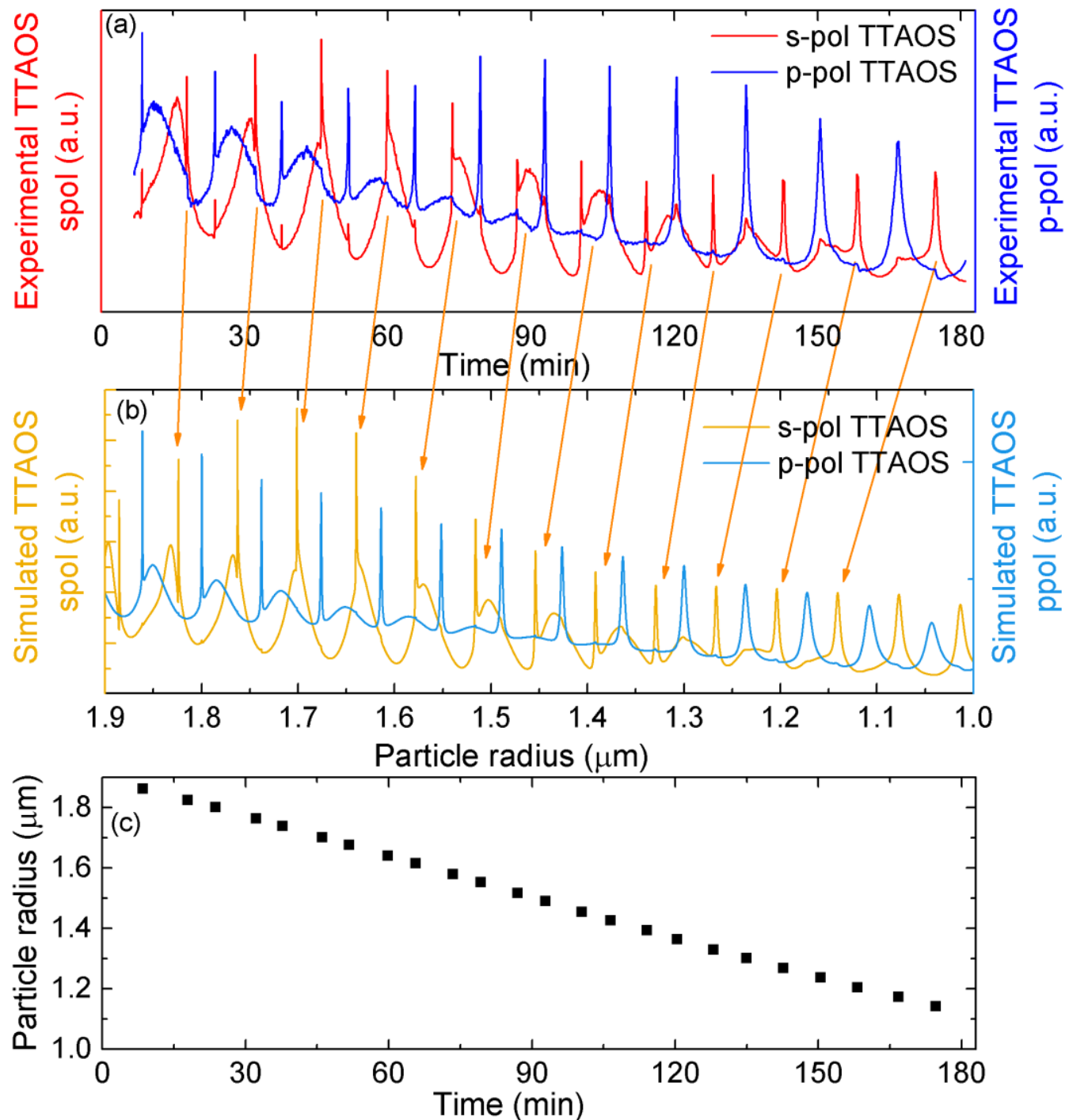

Figure A. 1. Experimental (a) and simulated (b) Total TAOS for both s- and p-pol. The assignment of the measured Total TAOS peaks to the simulated spectra is shown for the s-pol by arrows. (For sake of clarity, arrows for the same assignment for the p-pol peaks are not shown.) The sharp peaks indicated by the arrows are the ones used to retrieve the particle radius. Nonetheless the whole shape of each peak is crucial for the assignment. Panel (c) shows the time-evolution of the particle radius retrieved from this peak assignment.

\section{ACKNOWLEDGMENTS}

This work was supported by the Swiss National Science Foundation (SNSF grants no. 200020_172472 and 200020_177479) and the ETH Zurich (grant no. ETH-42 18-1). We would like to thank David Stapfer, Markus Steger and Timo Wey from the ETH mechanical and electronic shops for their help.

\section{REFERENCES}

[1] Pluschke, P. and Schleibinger, H., [Indoor air pollution] Springer (2018).

[2] Seinfeld, J. H. and Pandis, S. N., [Atmospheric chemistry and physics: from air pollution to climate change] John Wiley \& Sons (2012).

[3] Haywood, J. and Boucher, O. "Estimates of the direct and indirect radiative forcing due to tropospheric aerosols: A review". Rev. Geophys. 38, 513-543 (2000). 
[4] Lim, S. S., et al. "A comparative risk assessment of burden of disease and injury attributable to 67 risk factors and risk factor clusters in 21 regions, 1990-2010: a systematic analysis for the Global Burden of Disease Study 2010". The Lancet. 380, 2224-2260 (2012).

[5] Cammenga, H. K., Schulze, F. W. and Theuerl, W. "Vapor pressure and evaporation coefficient of glycerol". J. Chem. Eng. Data. 22, 131-134 (1977).

[6] Laskin, A., Laskin, J. and Nizkorodov, S. A. "Chemistry of Atmospheric Brown Carbon". Chem. Rev. 115, 4335$4382(2015)$.

[7] Diveky, M. E., Roy, S., Cremer, J. W., David, G. and Signorell, R. "Assessing relative humidity dependent photoacoustics to retrieve mass accommodation coefficients of single optically trapped aerosol particles". Phys. Chem. Chem. Phys. 21, 4721-4731 (2019).

[8] Bohren, C. F. and Huffman, D. R., [Absorption and Scattering of Light by Small Particles] Wiley (1983).

[9] Mishchenko, M. I., Travis, L. D. and Lacis, A. A., [Scattering, Absorption, and Emission of Light by Small Particles] Cambridge university press (2002).

[10] George, C., Ammann, M., D’Anna, B., Donaldson, D. J. and Nizkorodov, S. A. "Heterogeneous Photochemistry in the Atmosphere". Chem. Rev. 115, 4218-4258 (2015).

[11] Cwiertny, D. M., Young, M. A. and Grassian, V. H. "Chemistry and Photochemistry of Mineral Dust Aerosol". Annu. Rev. Phys. Chem. 59, 27-51 (2008).

[12] Banerjee, S., Gnanamani, E., Yan, X. and Zare, R. N. "Can all bulk-phase reactions be accelerated in microdroplets?". Analyst. 142, 1399-1402 (2017).

[13] Vortisch, H., Kramer, B., Weidinger, I., Woste, L., Leisner, T., Schwell, M., Baumgartel, H. and Ruhl, E. "Homogeneous freezing nucleation rates and crystallization dynamics of single levitated sulfuric acid solution droplets". Phys. Chem. Chem. Phys. 2, 1407-1413 (2000).

[14] Colberg, C. A., Krieger, U. K. and Peter, T. "Morphological Investigations of Single Levitated H2SO4/NH3/H2O Aerosol Particles during Deliquescence/Efflorescence Experiments". J. Phys. Chem. A. 108, 2700-2709 (2004).

[15] Esat, K., David, G., Poulkas, T., Shein, M. and Signorell, R. "Phase transition dynamics of single optically trapped aqueous potassium carbonate particles". Phys. Chem. Chem. Phys. 20, 11598-11607 (2018).

[16] Brunamonti, S., Krieger, U. K., Marcolli, C. and Peter, T. "Redistribution of black carbon in aerosol particles undergoing liquid-liquid phase separation". Geophys. Res. Lett. 42, 2532-2539 (2015).

[17] Veghte, D. P., Altaf, M. B. and Freedman, M. A. "Size Dependence of the Structure of Organic Aerosol". J. Am. Chem. Soc. 135, 16046-16049 (2013).

[18] Athanasiadis, A., et al. "Dynamic viscosity mapping of the oxidation of squalene aerosol particles". Phys. Chem. Chem. Phys. 18, 30385-30393 (2016).

[19] Nirmal, M., Dabbousi, B. O., Bawendi, M. G., Macklin, J. J., Trautman, J. K., Harris, T. D. and Brus, L. E. "Fluorescence intermittency in single cadmium selenide nanocrystals". Nature. 383, 802-804 (1996).

[20] Cremer, J. W., Thaler, K. M., Haisch, C. and Signorell, R. "Photoacoustics of single laser-trapped nanodroplets for the direct observation of nanofocusing in aerosol photokinetics". Nat. Commun. 7, 10941 (2016).

[21] Boucher, O. and Anderson, T. L. "General circulation model assessment of the sensitivity of direct climate forcing by anthropogenic sulfate aerosols to aerosol size and chemistry". J. Geophys. Res. 100, 26117-26134 (1995).

[22] Ray, A. K., Johnson, R. D. and Souyri, A. "Dynamic behavior of single glycerol droplets in humid air streams". Langmuir. 5, 133-140 (1989).

[23] David, G., Esat, K., Ritsch, I. and Signorell, R. "Ultraviolet broadband light scattering for optically-trapped submicron-sized aerosol particles". Phys. Chem. Chem. Phys. 18, 5477-5485 (2016).

[24] Cremer, J. W., Covert, P. A., Parmentier, E. A. and Signorell, R. "Direct Measurement of Photoacoustic Signal Sensitivity to Aerosol Particle Size". J. Phys. Chem. Lett. 8, 3398-3403 (2017).

[25] David, G., Esat, K., Thanopulos, I. and Signorell, R. "Digital holography of optically-trapped aerosol particles". Commun. Chem. 1, 46 (2018).

[26] David, G., Esat, K., Thanopulos, I. and Signorell, R., "Morphology and motion of single optically trapped aerosol particles from digital holography". Proc. SPIE,10723, (2018).

[27] Aptowicz, K. B., Pinnick, R. G., Hill, S. C., Pan, Y. L. and Chang, R. K. "Optical scattering patterns from single urban aerosol particles at Adelphi, Maryland, USA: A classification relating to particle morphologies". J. Geophys. Res. 111 (2006).

[28] Lu, J. W., Rickards, A. M. J., Walker, J. S., Knox, K. J., Miles, R. E. H., Reid, J. P. and Signorell, R. "Timescales of water transport in viscous aerosol: measurements on sub-micron particles and dependence on conditioning history". Phys. Chem. Chem. Phys. 16, 9819-9830 (2014). 